\title{
Differences in mandibular condyle and glenoid fossa morphology in relation to vertical and sagittal skeletal patterns: A cone-beam computed tomography study
}

\author{
Kyoung Jin Noh ${ }^{\mathrm{a}}$ (1) \\ Hyoung-Seon Baik \\ Sang-Sun $\mathrm{Han}^{\mathrm{c}}$ \\ Woowon Jang ${ }^{\mathrm{a}}$ \\ Yoon Jeong Choi ${ }^{\mathrm{b}}$ (1)
}

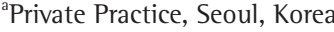

${ }^{b}$ Department of Orthodontics, The Institute of Craniofacial Deformity, Yonsei University College of Dentistry, Seoul, Korea

'Department of Oral and Maxillofacial Radiology, Yonsei University College of Dentistry, Seoul, Korea

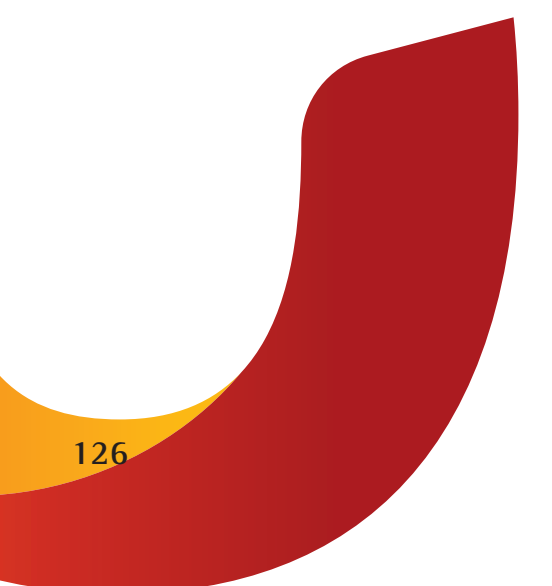

Objective: This study aimed to evaluate the following null hypothesis: there are no differences in the morphology of the temporomandibular joint (TMJ) structures in relation to vertical and sagittal cephalometric patterns. Methods: This retrospective study was performed with 131 participants showing no TMJ symptoms. The participants were divided into Class 1,11 , and 111 groups on the basis of their sagittal cephalometric relationships and into hyperdivergent, normodivergent, and hypodivergent groups on the basis of their vertical cephalometric relationships. The following measurements were performed using cone-beam computed tomography images and compared among the groups: condylar volume, condylar size (width, length, and height), fossa size (length and height), and condyle-to-fossa joint spaces at the anterior, superior, and posterior condylar poles. Results: The null hypothesis was rejected. The Class 111 group showed larger values for condylar width, condylar height, and fossa height than the Class 11 group $(p<0.05)$. Condylar volume and superior joint space in the hyperdivergent group were significantly smaller than those in the other two vertical groups $(p<0.001)$, whereas fossa length and height were significantly larger in the hyperdivergent group than in the other groups $(p<0.01)$. The hypodivergent group showed a greater condylar width than the hyperdivergent group $(p<0.01)$. The sagittal and vertical cephalometric patterns showed statistically significant interactions for fossa length and height. Conclusions: TMJ morphology differed across diverse skeletal cephalometric patterns. The fossa length and height were affected by the interactions of the vertical and sagittal skeletal patterns.

[Korean J Orthod 2021;51(2):126-134]

Key words: Temporomandibular joint, Cone-beam computed tomography, Cephalometrics

Received March 12, 2020; Revised September 21, 2020; Accepted November 16, 2020.

Corresponding author: Yoon Jeong Choi.

Associate Professor, Department of Orthodontics, The Institute of Craniofacial Deformity, Yonsei University College of Dentistry, 50-1 Yonsei-ro, Seodaemun-gu, Seoul 03722, Korea.

Tel +82-2-2228-3101 e-mail yoonjchoi@yuhs.ac

How to cite this article: Noh KJ, Baik HS, Han SS, Jang W, Choi YJ. Differences in mandibular condyle and glenoid fossa morphology in relation to vertical and sagittal skeletal patterns: A cone-beam computed tomography study. Korean J Orthod 2021;51:126-134.

(C) 2021 The Korean Association of Orthodontists.

This is an Open Access article distributed under the terms of the Creative Commons Attribution Non-Commercial License (http://creativecommons.org/licenses/by-nc/4.0) which permits unrestricted non-commercial use, distribution, and reproduction in any medium, provided the original work is properly cited. 


\section{INTRODUCTION}

The temporomandibular joint (TMJ) is an anatomical structure with significant implications in dental practice. The mandibular condyle is a part of the TMJ structure, and its shape and volume play important roles in the long-term stability of treatment outcomes in prosthodontic, orthodontic, and orthognathic patients. ${ }^{1,2}$ Therefore, dental practitioners should consider the morphology and positioning of the condyle throughout the treatment process.

The position and size of the mandibular condyle can be determined on the basis of the craniofacial morphology $^{3-9}$ and may be changed by temporomandibular disorder (TMD). ${ }^{10-14}$ The condyle and fossa are loaded differently in each individual because of individual differences in craniofacial morphology. ${ }^{4,5}$ These differences in loading result in different TMJ morphologies, ${ }^{15}$ indicating that the craniofacial morphology can influence the position and size of the condyle and glenoid fossa. Internal derangement of the TMJ can also affect the condyle. An anteriorly displaced disc may reduce the size of the mandibular condyle, which may not be recovered even after the TMD issues are resolved. ${ }^{10,13}$

The role of TMJ morphology as a diagnostic and prognostic indicator of orthodontic treatment has been investigated in relation to craniofacial features. ${ }^{8,16,17}$ The condyle exhibited a larger volume in patients with skeletal Class 111 malocclusion than in those with a Class 11 malocclusion..$^{5,7}$ Moreover, patients with a hyperdivergent facial profile showed a smaller volume and more superior position of the condyle than those with a hypodivergent profile. ${ }^{6,18}$ Nevertheless, the influences of the craniofacial skeletal patterns on TMJ morphology based on the interactive effects of the sagittal and vertical cephalometric relationships have not been comprehensively understood, although these interrelationships are responsible for various facial types. ${ }^{18,19}$

Several methods, such as conventional radiography, magnetic resonance imaging, and computed tomography (CT), have been used to examine the TMJ structure. ${ }^{3,5,12}$ Cone-beam CT (CBCT) has been recently introduced for measurements of bony structure since it produces high-resolution images with minimal distortion and less radiation dosage than conventional CT. ${ }^{11,20}$ Three-dimensional (3D) CBCT images allow measurements of volumes and lengths in multiple planes, which can contribute to accurate diagnoses and predictable treatment outcomes. ${ }^{2}$ The differences of TMJ morphology based on the interactive effects of the sagittal and vertical cephalometric relationships were barely investigated using 3D images. Therefore, this study aimed to evaluate the following null hypothesis: there are no differences in morphology of the TMJ structures in relation to vertical and sagittal cephalometric patterns.

\section{MATERIALS AND METHODS}

\section{Participants}

This retrospective study included 131 participants with no TMJ symptoms (48 men and 83 women; mean age, 23.5 years; range, 18.0-39.6 years) selected from 449 patients who had visited Yonsei University Dental Hospital between January 2012 and June 2016 and had undergone both lateral cephalography and CBCT including the TMJ structure. The participants had undergone CBCT for the following reasons: orthodontic diagnosis for orthognathic surgery; presence of impacted teeth; and evaluation of the available amount of alveolar bone for orthodontic tooth movement. The inclusion criteria were absence of signs and symptoms of TMD based on research diagnostic criteria for TMD; availability of lateral cephalography and СBCT images; and age over 18 years. The exclusion criteria were a history of orthodontic or orthognathic treatment; craniofacial skeletal deformity; and facial asymmetry with more than $4 \mathrm{~mm}$ of menton deviation. ${ }^{21}$

Using cephalometric analysis, the participants were classified on the basis of their vertical and sagittal skeletal relationships. The sella-nasion to mandibular plane (SN-MP) angle was used to divide the participants into hypodivergent $\left(\mathrm{SN}-\mathrm{MP}<30^{\circ}\right)$, normodivergent $\left(30^{\circ}\right.$ $\left.<\mathrm{SN}-\mathrm{MP}<38^{\circ}\right)$, and hyperdivergent $\left(\mathrm{SN}-\mathrm{MP}>38^{\circ}\right)$ groups, ${ }^{22}$ while the A point-nasion-B point (ANB) angle was used to divide them into Class $1\left(1^{\circ}<A N B<4^{\circ}\right)$, Class 11 (ANB $\left.>4^{\circ}\right)$, and Class 111 ( ANB $<1^{\circ}$ ) groups. ${ }^{19,23}$ Thus, the participants were eventually divided into nine subgroups (Table 1). On the basis of a previous study, ${ }^{12}$ we estimated that 12 participants per group were sufficient to detect differences in TMJ morphology among the three different groups. This study was approved by Yonsei University Dental Hospital Institutional Review Board (No. 2-2016-0001). Because of the retrospective nature of this study, the institutional review board waived the requirement for written informed patient consent.

\section{Measurements}

Three-dimensional images were acquired with a CBCT device (Alphard VEGA; ASAHI Roentgen IND, Kyoto, Japan) set at 5.0-8.0 mA and $80 \mathrm{kV}$, and images were captured for 17 seconds with a $0.30-\mathrm{mm}$ voxel size. The field of view was $154 \times 154 \mathrm{~mm}^{2}$. The images were transformed to the Digital Imaging and Communications in Medicine format, reconstructed, and analyzed with OnDemand software (Cybermed Inc., Seoul, Korea). The CBCT images were reoriented with the Frankfort horizontal (FH) plane parallel to the ground. Subsequently, 
Table 1. Demographic features of the participants

\begin{tabular}{|c|c|c|c|c|c|}
\hline & Group & $\begin{array}{l}\text { Hypodivergent } \\
\quad(\mathbf{n}=\mathbf{3 7})\end{array}$ & $\begin{array}{c}\text { Normodivergent } \\
\quad(n=47)\end{array}$ & $\begin{array}{l}\text { Hyperdivergent } \\
\quad(\mathrm{n}=47)\end{array}$ & $p$-value ${ }^{\dagger}$ \\
\hline \multirow[t]{4}{*}{ Age (yr) } & Class I $(n=43)$ & $\begin{array}{c}25.6 \pm 5.2 \\
(\mathrm{n}=11 ; \mathrm{M} 9, \mathrm{~F} 2)\end{array}$ & $\begin{array}{c}21.0 \pm 3.3 \\
(\mathrm{n}=16 ; \mathrm{M} 5, \mathrm{~F} 11)\end{array}$ & $\begin{array}{c}23.9 \pm 4.7 \\
(\mathrm{n}=16 ; \mathrm{M} 3, \mathrm{~F} 13)\end{array}$ & 0.93 \\
\hline & Class II $(\mathrm{n}=42)$ & $\begin{array}{c}27.6 \pm 8.3 \\
(\mathrm{n}=11 ; \mathrm{M} 4, \mathrm{~F} 7)\end{array}$ & $\begin{array}{c}23.4 \pm 5.9 \\
(\mathrm{n}=15 ; \mathrm{M} 5, \mathrm{~F} 10)\end{array}$ & $\begin{array}{c}23.0 \pm 6.0 \\
(\mathrm{n}=16 ; \mathrm{M} 4, \mathrm{~F} 12)\end{array}$ & 0.08 \\
\hline & Class III $(\mathrm{n}=46)$ & $\begin{array}{c}20.9 \pm 3.9 \\
(\mathrm{n}=15 ; \mathrm{M} 8, \mathrm{~F} 7)\end{array}$ & $\begin{array}{c}24.3 \pm 5.1 \\
(\mathrm{n}=16 ; \mathrm{M} 4, \mathrm{~F} 12)\end{array}$ & $\begin{array}{c}20.8 \pm 2.7 \\
(\mathrm{n}=15 ; \mathrm{M} 6, \mathrm{~F} 9)\end{array}$ & 0.08 \\
\hline & $p$-value* & 0.17 & 0.68 & 0.40 & \\
\hline \multirow[t]{4}{*}{$\operatorname{ANB}\left({ }^{\circ}\right)$} & Class I & $2.5 \pm 0.8$ & $2.9 \pm 1.0$ & $2.4 \pm 0.9$ & 0.44 \\
\hline & Class II & $5.7 \pm 1.2$ & $5.6 \pm 1.0$ & $6.8 \pm 1.6$ & 0.08 \\
\hline & Class III & $-1.8 \pm 3.3$ & $-2.5 \pm 2.8$ & $-2.0 \pm 1.4$ & 0.79 \\
\hline & $p$-value* & $0.00^{*}$ & $0.00^{*}$ & $0.00^{*}$ & \\
\hline \multirow[t]{4}{*}{ SN-MP $\left(^{\circ}\right)$} & Class I & $26.1 \pm 7.1$ & $34.2 \pm 2.2$ & $43.7 \pm 3.7$ & $0.00^{\dagger}$ \\
\hline & Class II & $25.4 \pm 2.4$ & $35.3 \pm 2.0$ & $44.5 \pm 3.4$ & $0.00^{\dagger}$ \\
\hline & Class III & $26.7 \pm 2.2$ & $34.3 \pm 2.2$ & $42.1 \pm 2.6$ & $0.00^{\dagger}$ \\
\hline & $p$-value ${ }^{*}$ & 0.87 & 0.33 & 0.16 & \\
\hline
\end{tabular}

Values are presented as mean \pm standard deviation.

ANB, A point-nasion-B point angle; SN-MP, sella-nasion to mandibular plane angle; M, male; F, female.

${ }^{*} p$-value indicates the analysis of variance (ANOVA) results for comparisons among the three sagittal (Class I, Class II, and Class III) groups, while ${ }^{\dagger} p$-value indicates the ANOVA results for comparisons among three vertical (hypodivergent, normodivergent, and hyperdivergent) groups.

${ }^{*} p<0.05 ;{ }^{\dagger} p<0.05$.
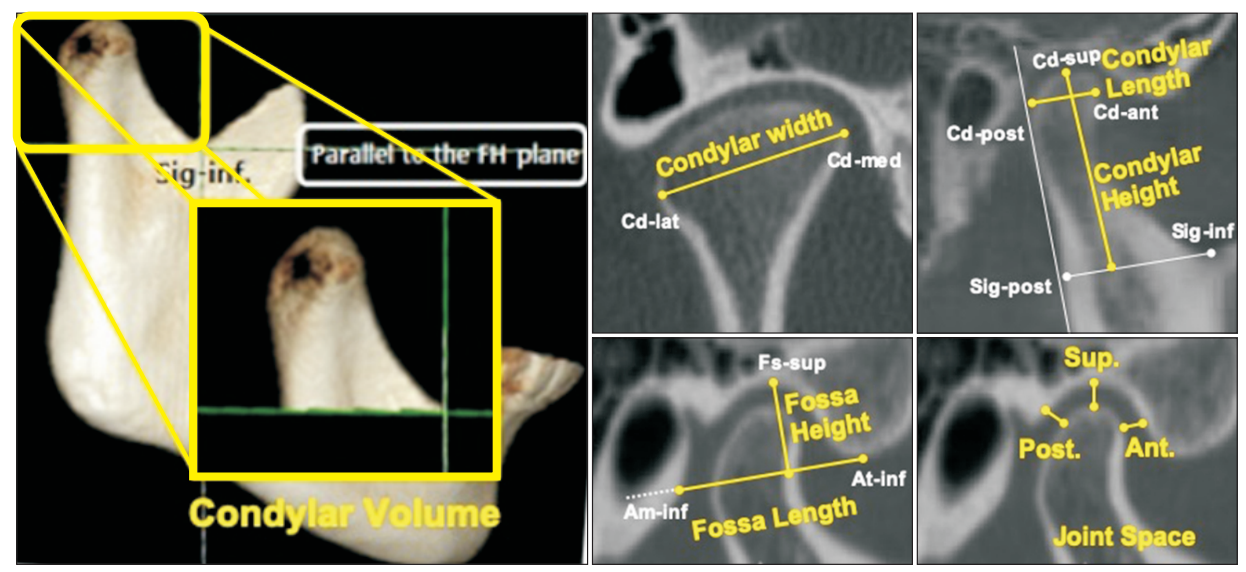

Figure 1. Measurements for the temporomandibular joint structure. Condylar volume was measured from a three-dimensional reconstructed image; condylar width was measured on the coronal section; and other measurements, including condylar length and height, fossa length and height, and superior, anterior, and posterior joint spaces, were measured on the same sagittal section. Please refer to Tables 2 and 3 for definitions of the abbreviations and measurements.

the midsagittal reference plane, which was perpendicular to the FH plane and passed through the nasion, was automatically set.

This study was designed to analyze condylar volume, condylar size (width, length, and height), fossa size (length and height), and condyle-to-fossa joint spaces at the anterior, superior, and posterior condylar poles (Figure 1). For measurements, we identified 10 anatomical landmarks (Cd-med, Cd-lat, Cd-sup, Cd-ant, Cdpost, Sig-inf, Sig-post, Fs-sup, At-inf, and Am-inf), as defined in Table 2 . The condylar volume was defined as the mandibular condyle bound inferiorly by a plane that 
Table 2. Definitions of the landmarks used in this study

\begin{tabular}{|c|c|c|}
\hline \multicolumn{2}{|c|}{ Landmark (abbreviation) } & \multirow{2}{*}{$\begin{array}{l}\text { Definition } \\
\text { The most medial point of the condylar head on the coronal section }\end{array}$} \\
\hline Condyle & Medial (Cd-med) & \\
\hline & Lateral (Cd-lat) & The most lateral point of the condylar head on the coronal section \\
\hline & Superior (Cd-sup) & $\begin{array}{l}\text { The most superior point of the condylar head identified on the axial and sagittal } \\
\text { sections }\end{array}$ \\
\hline & Anterior (Cd-ant) & $\begin{array}{l}\text { The most anterior point of the condylar head within a } 5 \mathrm{~mm} \text {-radius from Cd-sup } \\
\text { on the sagittal section }\end{array}$ \\
\hline & Posterior (Cd-post) & $\begin{array}{l}\text { The most posterior point of the condylar head within a } 5 \mathrm{~mm} \text {-radius from Cd-sup } \\
\text { on the sagittal section }\end{array}$ \\
\hline \multirow[t]{2}{*}{ Sigmoid } & Inferior (Sig-inf) & The most inferior point of the sigmoid notch \\
\hline & Posterior (Sig-post) & $\begin{array}{l}\text { Perpendicular point from Sig-inf to the tangent line of the ramal posterior surface } \\
\text { on the sagittal section }\end{array}$ \\
\hline \multicolumn{2}{|c|}{ Fossa superior (Fs-sup) } & $\begin{array}{l}\text { The point showing the shortest distance from Cd-sup to the superior wall of the } \\
\text { glenoid fossa }\end{array}$ \\
\hline \multicolumn{2}{|c|}{ Articular tubercle (At-inf) } & The most inferior point of the articular tubercle \\
\hline \multicolumn{2}{|c|}{ Auditory meatus (Am-inf) } & The most inferior point of the auditory meatus \\
\hline
\end{tabular}

Table 3. Definitions of the measurements performed in this study

\begin{tabular}{|c|c|c|}
\hline \multicolumn{2}{|c|}{ Measurement } & \multirow{2}{*}{$\begin{array}{l}\text { Definition } \\
\begin{array}{l}\text { Volume of the mandibular condyle bound inferiorly by a parallel plane to the FH plane and } \\
\text { passing through Sig-inf }\end{array}\end{array}$} \\
\hline Condyle & Volume* & \\
\hline & Width $^{\dagger}$ & Distance between Cd-med and Cd-lat \\
\hline & Length & Distance between Cd-ant and Cd-post \\
\hline & Height & Perpendicular distance from Cd-sup to the line between Sig-inf and Sig-post \\
\hline \multirow[t]{2}{*}{ Fossa } & Length & $\begin{array}{l}\text { Distance from At-inf to a point where the line connecting At-inf and Am-inf meets the posterior } \\
\text { wall of the glenoid fossa in the selected sagittal section }\end{array}$ \\
\hline & Height & $\begin{array}{l}\text { Perpendicular distance from Fs-sup to the line connecting At-inf and Am-inf in the selected } \\
\text { sagittal section }\end{array}$ \\
\hline \multirow[t]{3}{*}{ Joint space } & Superior & Distance from Cd-sup to Fs-sup \\
\hline & Anterior & The shortest distance from Cd-ant to the corresponding glenoid fossa \\
\hline & Posterior & The shortest distance from Cd-post to the corresponding glenoid fossa \\
\hline
\end{tabular}

All measurements were performed by using OnDemand software (Cybermed Inc., Seoul, Korea).

*Condylar volume was measured by separating the condylar structure from the mandible.

${ }^{\dagger}$ Condylar width was measured on the coronal section

Other parameters were measured on the sagittal section.

See Table 2 for definitions of each landmark.

passes through the Sig-inf point and is parallel to the FH plane ${ }^{24}$ (Table 3). From serial coronal sections, we selected one section that included the most medial and lateral points (Cd-med and Cd-lat, respectively) and consequently showed the widest distance of the condyle. On this coronal section, condylar width, which was the distance between $\mathrm{Cd}$-med and Cd-lat, was measured. For selection of the sagittal section, we identified the most superior point of the condyle (Cd-sup) from serial axial images, and then confirmed the landmark on the sagittal section as the most superior point of the condyle. ${ }^{8}$
The sagittal section that contained Cd-sup was finally selected and used for measurement of condylar length and height, ${ }^{24,25}$ fossa length and height, ${ }^{5}$ and three (superior, anterior, and posterior) joint spaces ${ }^{6}$ (Figure 1 and Table 3). The measurements were performed on both sides, and the mean values were used.

\section{Statistical analysis}

One examiner performed all measurements. To evaluate intra-examiner reliability, the same examiner re-analyzed 20 randomly selected participants within a 2 -week 
interval. Intraclass correlation coefficients of 0.995 were achieved, which confirmed acceptable reproducibility of the measurements.

Normal distribution of the data was confirmed by the Kolmogorov-Smirnov test. One-way analysis of variance (ANOVA) and Scheffe post-hoc test were used to compare Class 1,11 , and 111 groups according to the sagittal skeletal patterns. The same tests were performed to compare hypodivergent, normodivergent, and hyperdivergent groups according to the vertical skeletal patterns. The nine subgroups were compared by two-way ANOVA and Bonferroni post-hoc test to evaluate the interactions between the sagittal and vertical cephalometric patterns. All measurements were analyzed by using IBM SPSS Statistics software (ver. 23.0; IBM Corp.,
Armonk, NY, USA).

\section{RESULTS}

The three sagittal groups showed significant differences in condylar width, condylar height, and fossa height: the Class 111 group showed larger values of condylar width, condylar height, and fossa height than the Class 11 group ( $p<0.05$; Table 4). In contrast, condylar volume, condylar width, fossa height and length, and superior joint space showed significant differences among the three vertical groups $(p<0.01$; Table 5): condylar volume and superior joint space in the hyperdivergent group were significantly smaller than those in the other two vertical groups $(p<0.001)$, whereas fossa length

Table 4. Comparison of dimensions of temporomandibular joint structure according to sagittal skeletal patterns

\begin{tabular}{|c|c|c|c|c|c|}
\hline \multicolumn{2}{|c|}{ Variable } & Class I $(n=43)$ & Class II $(n=42)$ & Class III $(n=46)$ & $p$-value \\
\hline \multicolumn{2}{|c|}{ Condylar volume $\left(\mathrm{mm}^{3}\right)$} & $1,086.8 \pm 287.7$ & $1,096.2 \pm 283.23$ & $1,084.0 \pm 271.8$ & 0.978 \\
\hline \multirow[t]{3}{*}{ Condyle } & Width (mm) & $16.1 \pm 3.6^{\mathrm{ab}}$ & $15.4 \pm 3.1^{\mathrm{a}}$ & $17.1 \pm 2.7^{\mathrm{b}}$ & $0.037^{*}$ \\
\hline & Length (mm) & $7.5 \pm 1.2$ & $7.6 \pm 1.3$ & $7.4 \pm 1.0$ & 0.629 \\
\hline & Height (mm) & $23.2 \pm 4.0^{\mathrm{ab}}$ & $21.9 \pm 2.9^{\mathrm{a}}$ & $25.2 \pm 3.9^{\mathrm{b}}$ & $0.000^{* * *}$ \\
\hline \multirow[t]{2}{*}{ Fossa } & Length (mm) & $20.3 \pm 2.2$ & $20.3 \pm 2.0$ & $20.7 \pm 2.0$ & 0.666 \\
\hline & Height $(\mathrm{mm})$ & $9.3 \pm 1.7^{\mathrm{ab}}$ & $8.7 \pm 1.5^{\mathrm{a}}$ & $9.7 \pm 1.4^{\mathrm{b}}$ & $0.011^{*}$ \\
\hline \multirow[t]{3}{*}{ Joint space } & Superior (mm) & $3.0 \pm 0.8$ & $2.9 \pm 0.9$ & $2.9 \pm 0.8$ & 0.598 \\
\hline & Anterior (mm) & $2.6 \pm 0.6$ & $2.9 \pm 0.7$ & $2.7 \pm 0.8$ & 0.305 \\
\hline & Posterior (mm) & $2.6 \pm 0.8$ & $2.5 \pm 0.9$ & $2.5 \pm 0.7$ & 0.765 \\
\hline
\end{tabular}

Values are presented as mean \pm standard deviation.

$p$-value indicates one-way analysis of variance results for comparisons among the three sagittal groups. The same letters indicate that there were no statistically significant differences.

${ }^{*} p<0.05 ;{ }^{* * *} p<0.001$.

Table 5. Comparison of dimensions of temporomandibular joint structure according to vertical skeletal patterns

\begin{tabular}{|c|c|c|c|c|c|}
\hline \multicolumn{2}{|c|}{ Variable } & $\begin{array}{l}\text { Hypodivergent } \\
(\mathbf{n}=\mathbf{3 7})\end{array}$ & $\begin{array}{l}\text { Normodivergent } \\
(n=47)\end{array}$ & $\begin{array}{c}\text { Hyperdivergent } \\
(\mathrm{n}=47)\end{array}$ & $p$-value \\
\hline \multicolumn{2}{|c|}{ Condylar volume $\left(\mathrm{mm}^{3}\right)$} & $1152.4 \pm 275.1^{\mathrm{a}}$ & $1191.3 \pm 279.2^{\mathrm{a}}$ & $936.4 \pm 211.7^{\mathrm{b}}$ & $0.000^{* * *}$ \\
\hline \multirow[t]{3}{*}{ Condyle } & Width (mm) & $17.6 \pm 3.0^{\mathrm{a}}$ & $16.1 \pm 2.8^{\mathrm{ab}}$ & $15.2 \pm 3.5^{\mathrm{b}}$ & $0.003^{* *}$ \\
\hline & Length (mm) & $7.6 \pm 1.2$ & $7.7 \pm 1.2$ & $7.2 \pm 1.1$ & 0.104 \\
\hline & Height (mm) & $23.1 \pm 3.3$ & $24.2 \pm 4.1$ & $23.1 \pm 4.0$ & 0.292 \\
\hline \multirow[t]{2}{*}{ Fossa } & Length (mm) & $20.1 \pm 2.1^{\mathrm{a}}$ & $20.0 \pm 1.7^{\mathrm{a}}$ & $21.2 \pm 2.1^{\mathrm{b}}$ & $0.007^{* *}$ \\
\hline & Height (mm) & $8.5 \pm 1.2^{\mathrm{a}}$ & $9.0 \pm 1.6^{\mathrm{a}}$ & $10.1 \pm 1.5^{\mathrm{b}}$ & $0.000^{* * *}$ \\
\hline \multirow[t]{3}{*}{ Joint space } & Superior (mm) & $3.3 \pm 0.8^{\mathrm{a}}$ & $3.0 \pm 0.9^{\mathrm{a}}$ & $2.6 \pm 0.7^{\mathrm{b}}$ & $0.000^{* * *}$ \\
\hline & Anterior (mm) & $2.7 \pm 0.6$ & $2.7 \pm 0.7$ & $2.8 \pm 0.8$ & 0.861 \\
\hline & Posterior (mm) & $2.4 \pm 0.7$ & $2.7 \pm 0.9$ & $2.5 \pm 0.8$ & 0.280 \\
\hline
\end{tabular}

Values are presented as mean \pm standard deviation.

$p$-value indicates one-way analysis of variance results for comparisons among the three vertical groups. The same letters indicate that there were no statistically significant differences.

${ }^{* *} p<0.01 ;{ }^{* * *} p<0.001$. 
and height were significantly higher in the hyperdivergent group than in the other groups $(p<0.01)$. With respect to the condylar width, the hypodivergent group showed a wider condylar head than the hyperdivergent group $(p<0.01)$.

Two-way ANOVA tests among the nine subgroups showed no statistically significant interactions between the sagittal and vertical cephalometric patterns for the condylar parameters and joint spaces $(p>0.05)$. However, statistically significant interactions were observed between the sagittal and vertical cephalometric patterns for fossa length and height ( $p<0.05$; Table 6 and Figure 2): for fossa length, the Class III-hyperdivergent subgroup showed a higher value than the other subgroups, and for fossa height, the Class 1-hyperdivergent, Class III-hyperdivergent, and Class III-normodivergent subgroups showed higher values than the other subgroups.

\section{DISCUSSION}

This study aimed to compare the morphology of TMJ structures and the spatial relationships between the condyle and fossa in relation to vertical and sagittal cephalometric patterns, and the findings suggested that the Class 111 group showed higher values for condylar width, condylar height, and fossa height than the Class 11 group. Condylar volume, condylar width, and superior joint space in the hyperdivergent group were significantly smaller than those in the hypodivergent group, while fossa length and height were significantly larger in the hyperdivergent group than the hypodivergent group. In addition, there were statistically significant interactions between the vertical and sagittal cephalometric patterns in fossa length and height. Therefore, the null hypothesis of this study was rejected.

Table 6. Interaction effect between vertical and sagittal skeletal patterns for fossa length and height

\begin{tabular}{|c|c|c|c|c|c|}
\hline Variable & & Class I $(n=43)$ & Class II $(n=42)$ & Class III $(n=46)$ & $p$-value \\
\hline \multirow[t]{4}{*}{ Fossa length (mm) } & Hypodivergent $(\mathrm{n}=37)$ & $20.8 \pm 2.8^{\mathrm{a}}$ & $19.8 \pm 1.2^{\mathrm{a}}$ & $19.8 \pm 2.1^{\mathrm{a}}$ & 0.470 \\
\hline & Normodivergent $(n=47)$ & $19.9 \pm 2.0^{\mathrm{a}}$ & $20.2 \pm 2.0^{\mathrm{a}}$ & $19.9 \pm 1.1^{\mathrm{a}}$ & 0.869 \\
\hline & Hyperdivergent $(\mathrm{n}=47)$ & $20.4 \pm 1.9^{\mathrm{a}}$ & $20.8 \pm 2.4^{\mathrm{ab}}$ & $22.4 \pm 1.5^{\mathrm{b}}$ & $0.019 *$ \\
\hline & $p$-value & 0.578 & 0.423 & $0.000^{*}$ & $0.045^{\dagger}$ \\
\hline \multirow[t]{4}{*}{ Fossa height (mm) } & Hypodivergent $(\mathrm{n}=37)$ & $8.6 \pm 1.4^{\mathrm{a}}$ & $8.5 \pm 1.5^{\mathrm{ac}}$ & $8.5 \pm 0.7^{\mathrm{a}}$ & 0.928 \\
\hline & Normodivergent $(n=47)$ & $8.3 \pm 1.4^{\mathrm{a}}$ & $8.3 \pm 1.4^{\mathrm{ac}}$ & $10.2 \pm 1.2^{\mathrm{b}}$ & $0.000^{*}$ \\
\hline & Hyperdivergent $(\mathrm{n}=47)$ & $10.7 \pm 1.2^{\mathrm{d}}$ & $9.2 \pm 1.6^{\mathrm{ac}}$ & $10.4 \pm 1.4^{\mathrm{bcd}}$ & $0.012^{*}$ \\
\hline & $p$-value & $0.000^{*}$ & 0.194 & $0.000^{*}$ & $0.002^{\dagger}$ \\
\hline
\end{tabular}

Values are presented as mean \pm standard deviation.

Two-way analysis of variance (ANOVA) was performed to investigate the interaction between two variables.

${ }^{*} p$-value indicates statistically significant differences within one variable (either vertical or sagittal pattern), while ${ }^{\dagger} p$-value indicates statistically significant interactions between sagittal and vertical skeletal patterns by two-way ANOVA. The same letters indicate that there were no statistically significant differences.
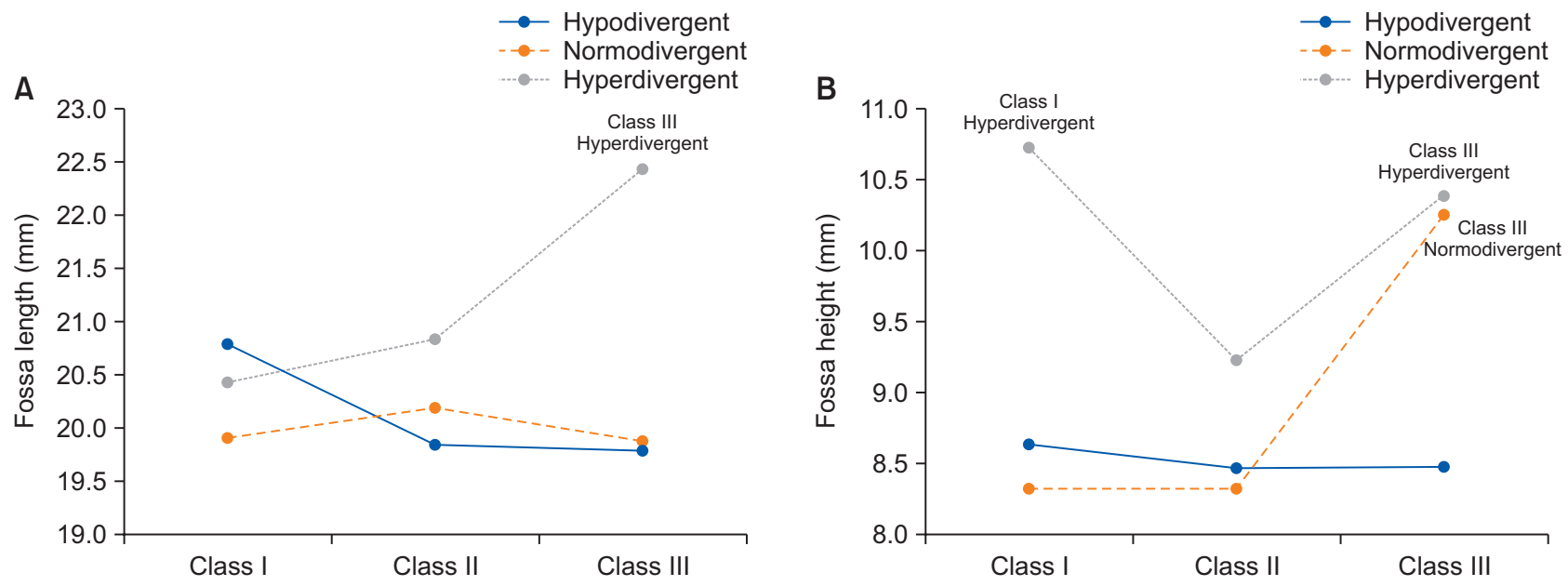

Figure 2. Interaction between vertical and sagittal skeletal patterns and fossa length (A) and height (B). 
The TMJ structure is affected by sagittal or vertical craniofacial morphology. The condylar width and height were larger in the Class 111 group than in the Class 11 group while the condylar volume was not significantly different between the groups. This finding indicates that the condylar width and height may result from excessive vertical growth of the mandibular ramus in Class 111 malocclusion. ${ }^{5}$ The condylar volume is not affected by sagittal jaw position but is influenced by the vertical cephalometric relationship: the hyperdivergent group showed a significantly smaller condylar volume than the other vertical groups. The hyperdivergent group also showed a smaller condylar width, larger fossa height and length, and smaller superior joint space than the other facial profiles. Although a recent study reported different findings, showing that the condylar width and fossa height were not significantly different among different vertical cephalometric relationships in adolescents, ${ }^{18}$ the differences in participants' ages and the numbers and definitions of each landmark might have been responsible for the differences. The sagittal growth at the condyle in the hyperdivergent profile ${ }^{26}$ which leads to backward growth rotation of the mandible and backward inclination of the condylar head, ${ }^{27}$ may cause a superiorly positioned condyle and a subsequent small superior joint space since preadolescence. ${ }^{6,18,28}$ The smaller superior joint space in the hyperdivergent profile than in the hypodivergent profile was confirmed in other studies, which suggested the possibility of increased anterior facial height because of reduced condylar growth potential. ${ }^{4,6,18,28}$

The fossa morphology is influenced by the interaction of sagittal and vertical cephalometric patterns. The fossa length showed a statistically significant difference among vertical patterns but not among sagittal patterns. However, in analyses combining the vertical and sagittal patterns, the Class III-hyperdivergent subgroup showed a larger fossa length than the other subgroups (Figure 2A). Although the Class 111 group has been reported to show a larger fossa length than the Class 11 group, ${ }^{4,5}$ the growth of the fossa length appears to be accelerated not only by Class 111 malocclusion but also by the hyperdivergent profile.

The fossa height is also influenced by both vertical and sagittal cephalometric patterns. When compared separately, the fossa height did not show any differences among different facial patterns ${ }^{18}$ or increased as the mandible was positioned forward and the facial divergence increased. However, when analyzed together, the differences in the fossa height based on the vertical pattern were larger than those based on the sagittal pattern: the hyperdivergent subgroups showed larger fossa heights than the hypodivergent and normodivergent subgroups, except the Class 111-normodivergent subgroup (Figure 2B). Since the fossa height is thought to be related to functional demands such as condylar shape $^{15}$ or anterior mandibular movement, ${ }^{29}$ the facial divergence may represent more functional demands than the sagittal cephalometric pattern.

The large condyle provides stable support for occlusal changes and is considered more resistant to displacement because the fossa and condyle are well-fitted. Conversely, the small condyle may provide unreliable support for occlusal changes and can be easily displaced because it makes stable fitting of the components difficult. ${ }^{5,30}$ Thus, it is advisable for practitioners to pay attention to the condylar condition of each patient before initiating treatment that may lead to any changes in the occlusion, especially for patients with a small condyle or large fossa, such as those with a hyperdivergent facial profile.

This study had some limitations. The articular disc could not be evaluated because only CBCT images were used. Therefore, asymptomatic patients with internal derangement may have been included in this study. To exclude TMD patients with disc displacement, the information obtained from additional diagnostic tools, such as magnetic resonance imaging examinations, may be necessary in future studies. The transverse skeletal condition, which can affect the malocclusion pattern, was not considered in this study, although the sagittal and vertical skeletal patterns of patients were assessed. Moreover, the number of patients was insufficient due to the limitations of the retrospective study design. This limitation can be overcome by a well-organized prospective study.

\section{CONCLUSION}

1. TMJ morphology differed according to diverse skeletal patterns: the findings showed significant differences in condylar width, condylar height, and fossa height among the three sagittal groups, while condylar volume, condylar width, fossa height and length, and superior joint space were significantly different among the three vertical groups.

2. The fossa length and height were affected by the interaction of the vertical and sagittal skeletal patterns.

3. For patients with a small condyle or large fossa, such as those with a hyperdivergent facial profile, it is advisable for practitioners to pay attention to the condylar condition before initiating treatment.

\section{CONFLICTS OF INTEREST}

No potential conflict of interest relevant to this article was reported. 


\section{ACKNOWLEDGEMENTS}

This research was supported by Korean Association of Orthodontists Research Fund, 2016.

\section{REFERENCES}

1. Krisjane Z, Urtane 1, Krumina G, Bieza A, Zepa K, Rogovska 1. Condylar and mandibular morphological criteria in the 2D and 3D MSCT imaging for patients with Class 11 division 1 subdivision malocclusion. Stomatologija 2007;9:67-71.

2. Tecco S, Saccucci M, Nucera R, Polimeni A, Pagnoni M, Cordasco G, et al. Condylar volume and surface in Caucasian young adult subjects. BMC Med Imaging 2010;10:28.

3. Katsavrias EG. Morphology of the temporomandibular joint in subjects with Class 11 Division 2 malocclusions. Am J Orthod Dentofacial Orthop 2006; 129:470-8.

4. Katsavrias EG, Halazonetis DJ. Condyle and fossa shape in Class 11 and Class 111 skeletal patterns: a morphometric tomographic study. Am J Orthod Dentofacial Orthop 2005;128:337-46.

5. Krisjane Z, Urtane 1, Krumina G, Zepa K. Threedimensional evaluation of TMJ parameters in Class 11 and Class 111 patients. Stomatologija 2009;11:32-6.

6. Park IY, Kim JH, Park YH. Three-dimensional conebeam computed tomography based comparison of condylar position and morphology according to the vertical skeletal pattern. Korean J Orthod 2015;45: 66-73.

7. Saccucci M, D’Attilio M, Rodolfino D, Festa F, Polimeni A, Tecco S. Condylar volume and condylar area in class 1, class 11 and class 111 young adult subjects. Head Face Med 2012;8:34.

8. Saccucci M, Polimeni A, Festa F, Tecco S. Do skeletal cephalometric characteristics correlate with condylar volume, surface and shape? A 3D analysis. Head Face Med 2012;8:15.

9. Vitral RW, Telles Cde S, Fraga MR, de Oliveira RS, Tanaka OM. Computed tomography evaluation of temporomandibular joint alterations in patients with class 11 division 1 subdivision malocclusions: condyle-fossa relationship. Am J Orthod Dentofacial Orthop 2004;126:48-52.

10. Kurita H, Uehara S, Yokochi M, Nakatsuka A, Kobayashi $\mathrm{H}$, Kurashina K. A long-term follow-up study of radiographically evident degenerative changes in the temporomandibular joint with different conditions of disk displacement. Int J Oral Maxillofac Surg 2006;35:49-54.

11. Sümbüllü MA, Cağlayan F, Akgül HM, Yilmaz AB. Radiological examination of the articular eminence morphology using cone beam CT. Dentomaxillofac Radiol 2012;41:234-40.

12. Yamada K, Tsuruta A, Hanada K, Hayashi T. Morphology of the articular eminence in temporomandibular joints and condylar bone change. J Oral Rehabil 2004;31:438-44.

13. Hasegawa H, Saitoh I, Nakakura-Ohshima K, Shigeta K, Yoshihara T, Suenaga S, et al. Condylar shape in relation to anterior disk displacement in juvenile females. Cranio 2011;29:100-10.

14. Paknahad M, Shahidi S, Akhlaghian M, Abolvardi M. ls mandibular fossa morphology and articular eminence inclination associated with temporomandibular dysfunction? J Dent (Shiraz) 2016;17:134-41.

15. Weinmann JP, Sicher H. Bone and bones: fundamentals of bone biology. St. Louis: Mosby; 1947.

16. Mupparapu M, Chow 1, Uppal A. Hard tissue structural changes in TMJ morphology prior to orthodontic therapy: a complex motion tomographic study. Quintessence lnt 2011;42:427-34.

17. Al-Riyami S, Moles DR, Cunningham SJ. Orthognathic treatment and temporomandibular disorders: a systematic review. Part 1. A new quality-assessment technique and analysis of study characteristics and classifications. Am J Orthod Dentofacial Orthop 2009;136:624.e1-15; discussion 624-5.

18. Chae JM, Park JH, Tai K, Mizutani K, Uzuka S, Miyashita W, et al. Evaluation of condyle-fossa relationships in adolescents with various skeletal patterns using cone-beam computed tomography. Angle Orthod 2020;90:224-32.

19. Kim JY, Lee SJ, Kim TW, Nahm DS, Chang Yl. Classification of the skeletal variation in normal occlusion. Angle Orthod 2005;75:311-9.

20. Petersson A. What you can and cannot see in TMJ imaging--an overview related to the RDC/TMD diagnostic system. J Oral Rehabil 2010;37:771-8.

21. Nur RB, Çakan DG, Arun T. Evaluation of facial hard and soft tissue asymmetry using cone-beam computed tomography. Am J Orthod Dentofacial Orthop 2016;149:225-37.

22. Kim SJ, Kim KH, Yu HS, Baik HS. Dentoalveolar compensation according to skeletal discrepancy and overjet in skeletal Class 111 patients. Am J Orthod Dentofacial Orthop 2014;145:317-24.

23. Kim JH, Gansukh 0, Amarsaikhan B, Lee SJ, Kim TW. Comparison of cephalometric norms between Mongolian and Korean adults with normal occlusions and well-balanced profiles. Korean J Orthod 2011;41:42-50.

24. Al-koshab M, Nambiar P, John J. Assessment of condyle and glenoid fossa morphology using CBCT in South-East Asians. PLoS One 2015;10:e0121682.

25. Hilgers ML, Scarfe WC, Scheetz JP, Farman AG. Ac- 
curacy of linear temporomandibular joint measurements with cone beam computed tomography and digital cephalometric radiography. Am J Orthod Dentofacial Orthop 2005;128:803-11.

26. Bjork A. Variations in the growth pattern of the human mandible: longitudinal radiographic study by the implant method. J Dent Res 1963;42Pt 2:400-11.

27. Björk A. Prediction of mandibular growth rotation. Am J Orthod 1969;55:585-99.

28. Burke G, Major P, Glover K, Prasad N. Correlations between condylar characteristics and facial morphology in Class 11 preadolescent patients. Am J Orthod Dentofacial Orthop 1998;114:328-36.

29. Cohlmia JT, Ghosh J, Sinha PK, Nanda RS, Currier GF. Tomographic assessment of temporomandibular joints in patients with malocclusion. Angle Orthod 1996;66:27-35.

30. Arnett GW, Gunson MJ. Facial planning for orthodontists and oral surgeons. Am J Orthod Dentofacial Orthop 2004;126:290-5. 